\title{
Compact UWB Monopole for Multilayer Applications
}

\author{
B. Sanz-Izquierdo, P.R. Young, Q. Bai and J.C. Batchelor
}

This paper is a postprint of a paper submitted to and accepted for publication in IET Microwaves, Antennas and Propagation and is subject to Institution of Engineering and Technology Copyright. The copy of record is available at IET Digital Library 


\title{
Compact UWB Monopole for Multilayer Applications
}

\author{
B. Sanz-Izquierdo, P.R. Young, Q. Bai and J.C. Batchelor \\ Department of Electronics, The University of Kent \\ Canterbury, Kent, CT2 7NT, UK. Tel: +44 1227827004 , Fax: +44 1227456084 \\ b.sanz@kent.ac.uk, p.r.young@kent.ac.uk, j.c.batchelor@kent.ac.uk
}

\begin{abstract}
:
A novel compact, dual layer UWB monopole antenna is presented. This low profile ultrawideband antenna is fed by a $50 \Omega$ shielded strip-line with an array of metal vias making the conducting walls. A printed disc monopole with a circular cut is the radiating element. The dual layer, shielded strip line feed allows for integration in multilayer technologies. The ultra-wideband, monopole characteristics of the antenna are confirmed experimentally.
\end{abstract}

\section{Introduction:}

Ultrawide-band communications systems have been investigated and developed over the last few decades. The technology promises high-speed transmission rate for short-range, indoor data communications. In 2002, the Federal communications Commission (FCC) allocated the spectrum from 3.1 to $10.6 \mathrm{GHz}$ for unlicensed UWB measurement, medical and communication applications [1]. The frequency allocation has prompted the development of antennas and systems capable of covering the complete UWB frequency band.

For ultra-wideband applications the dispersion in the transmission line medium of the system is of particular importance. Conventionally microstrip lines are used for RF and microwave circuit design. However, the hybrid nature of microstrip results in quasi-TEM propagation and therefore unwanted dispersion. Furthermore, microstrip lines are open 
structures which suffer from cross-talk and radiation losses. An attractive alternative to microstrip is shielded stripline which supports TEM mode propagation and is therefore non-dispersive. If the stripline is edge shielded then the problems of feed cross-talk and losses are reduced allowing highly integrated systems to be produced. The multilayer geometry of stripline is well suited to technologies such as microwave laminates, photoimageable thick-film and LTCC (Low Temperature Co-fired Ceramic) [6] which are becoming popular fabrication techniques as compact multilayer solutions based on stripline are easily manufactured.

Antennas have been proposed for ultrawide-band applications in three dimensional [2], and planar form [3], [4]. Planar monopole antennas have been developed with different shapes such as circular [4] and half disc [5].

This letter proposes a shielded strip-line fed UWB antenna design which can be integrated into multilayer circuit devices. The walls of the shielded strip line are formed using metal vias. The antenna is derived from the simple disc of [4] and consists of a disc with a circular edge cut, Fig.1. The new design is smaller in size than [4] when fabricated on the same permittivity substrate. Also, the feedline is better isolated from radiation and other circuitry when realized as shielded stripline.

\section{Antenna design:}

Figure 1 shows the proposed compact, UWB antenna for multilayer applications. The antenna was designed to cover the UWB frequencies 3.1 to $10.6 \mathrm{GHz}$ and was fabricated using RT Duroid substrate with a dielectric constant of 2.33. The multilayer structure consisted of two layers of thickness $1.575 \mathrm{~mm}$ with metal etched on the faces of each substrate. The rectangular shape of the ground plane was produced by etching the top and bottom metal clad of the top and bottom layer respectively. The antenna was fed by a $50 \mathrm{ohm}$ shielded strip-line. The stripline ground planes were terminated in the radiation region above and below the monopole disc which was located between the 2 substrate layers. The side walls of the shielded strip transmission line were fabricated using two arrays of metallised vias of diameter $0.5 \mathrm{~mm}$ and pitch $1 \mathrm{~mm}$. The separation between the two via arrays was $7.5 \mathrm{~mm}$. A tapered transition was used between the radiating element and the transmission line to provide good matching over the UWB bandwidth. The total 
dimensions of the antenna were $20 \times 35.5 \mathrm{~mm}$ and the other dimensions of the antennas are shown in table 1. For the same substrate permittivity, the new antenna has a surface area $70 \%$ smaller than the original antenna described in [4].

\section{Antenna analysis and results:}

Fig 3. Shows the simulated and measured return loss (S11) of the compact UWB antenna. The measured return loss was obtained using an HP 8722 ES network analyzer with coaxial calibration and CST Microwave Studio was used for the simulations. The calculated and measured $-10 \mathrm{~dB} \mathrm{S11}$ bandwidth achieves more than the $3-10.6 \mathrm{GHz}$ needed for the UWB communication systems.

A further analysis was carried out to determine the effect of antenna parameters on the input matched bandwidth and results are plotted in Fig.4 and 5. The antenna parameters studied were those most likely to be modified in the case of integrating the antenna with other multilayer devices. It was observed that the input match was sensitive to changes in the gap between the stripline ground planes and the circular radiating element and this is consistent with the experience reported in [4] for the coplanar monopole design. Altering the radius of the circular element, $r$, caused variations in the matching of the three main resonant modes with a larger disc reducing the lowest matched frequency and increasing the highest. The distance, $D$, between the main radiating disc and the cutting circle centre was used for fine tuning and to reduce the size of the antenna while preserving all the modes.

The measured $\mathrm{H}$ plane radiation pattern in the $x-z$ direction at 3.5, 6.0 and $9.0 \mathrm{GHz}$ are shown in Fig.5. Omni directional patterns were observed for this plane and there were no nulls deeper than $8 \mathrm{~dB}$ observed at any frequency. The measured peak gain across the band had a minimum value of $-1 \mathrm{dBi}$ at $3 \mathrm{GHz}$ and a maximum of $3.3 \mathrm{dBi}$ at $6 \mathrm{GHz}$.

\section{Conclusions:}

A reduced size ultra-wideband antenna intended for multilayer applications has been proposed in this paper. The input matched bandwidth for the novel antenna covers the 3 to $10.6 \mathrm{GHz}$ necessary for UWB applications. The antenna shows monopole radiation patterns with omni-directional patterns in the $x-z$ plane. The dual layer, strip line feeding 
characteristics of the antenna design allows for further design and implementation in other multilayer device technologies such us LTCC.

\section{ACKNOWLEDGEMENT}

The authors would like to thank Nigel Bourner, and Clive Birch for assistance with the fabrication and Nigel Simpson and Simon Jakes for help with the measurement of the antennas.

\section{REFERENCES}

[1] First Report and Order, Revision of Part 15 of the Commission's Rule Regarding Ultra Wide Band Transmission Systems”, Fed. Commun. Comm. FCC 02-48. Apr. 22, 2002

[2] K.-L. Wong, Y.-W. Chi and C.-H.Wu , "Wideband tri-plate monopole antenna", Electron. Lett. $25^{\text {th }}$ November 2004, Vol.40 N 24

[3] Jianxin Liang, Lu Guo, Choo C.Chiau and Xiadong Che, "CPW-Fed Circular Monopole Antenna for UWB Applications”, 2005 IEEE International Workshop on Antennas Technology, pp.

[4] Seong-Youp Suh, Warren L. Stutzman, and William A.Davis, “A New Ultrawideband Printed Monopole Antenna: The Planar Inverted Cone Antenna (PICA)” IEEE Trans. Antennas Propagat., vol.52 (5), MAY 2004.

[5] Taeyoung Yang and William A.Davis "Planar Half-Disk Antenna Structures for Ultra-Wideband Communications”, IEEE Antennas and Propagation Society International Symposium, vol.3, pp 2508-2511, 2004

[6] Rao R. Tummala and Joy Laskar "Gigabit Wireless: System-on-a-Package Techonoly", Proceedings of the IEEE, Vol. 92, No.2, February 2004 


\section{List of Figure captions:}

Figure 1. Geometry of the Dual-layer UWB antenna

Figure 2. Simulation and measured return loss (S11) of the dual-layer UWB antenna

Figure 3. Simulated S1 1 of the multilayer UWB antenna changing the radius (r) of the circular radiating element.

Figure 4. Simulated S11 of the multilayer UWB antenna changing distance from the centre of the radiating disc to the center of the cutting circle (D)

Figure 5. Measured $\mathrm{H}$ plane radiation pattern in the $x-z$ plane at $3.5 \mathrm{GHz}$ (dashed line), $6 \mathrm{GHz}$ (black), $9 \mathrm{GHz}$ (grey).

Table caption:

Table I. Dimensions of the multilayer Strip-line fed UWB antenna 


\begin{tabular}{|c|c|c|c|}
\hline Parameter & Value $(\mathrm{mm})$ & Parameter & Value $(\mathrm{mm})$ \\
\hline $\mathrm{Wg}$ & 20.0 & $\mathrm{r}$ & 7.5 \\
\hline $\mathrm{Ls}$ & 35.5 & $\mathrm{rc}$ & 7.5 \\
\hline $\mathrm{Lg}$ & 20.0 & $\mathrm{Lc}$ & 29.0 \\
\hline $\mathrm{Ld}$ & 1.5 & $\mathrm{D}$ & 13.0 \\
\hline
\end{tabular}

Table I. Dimensions of the multilayer Strip-line fed UWB antenna 


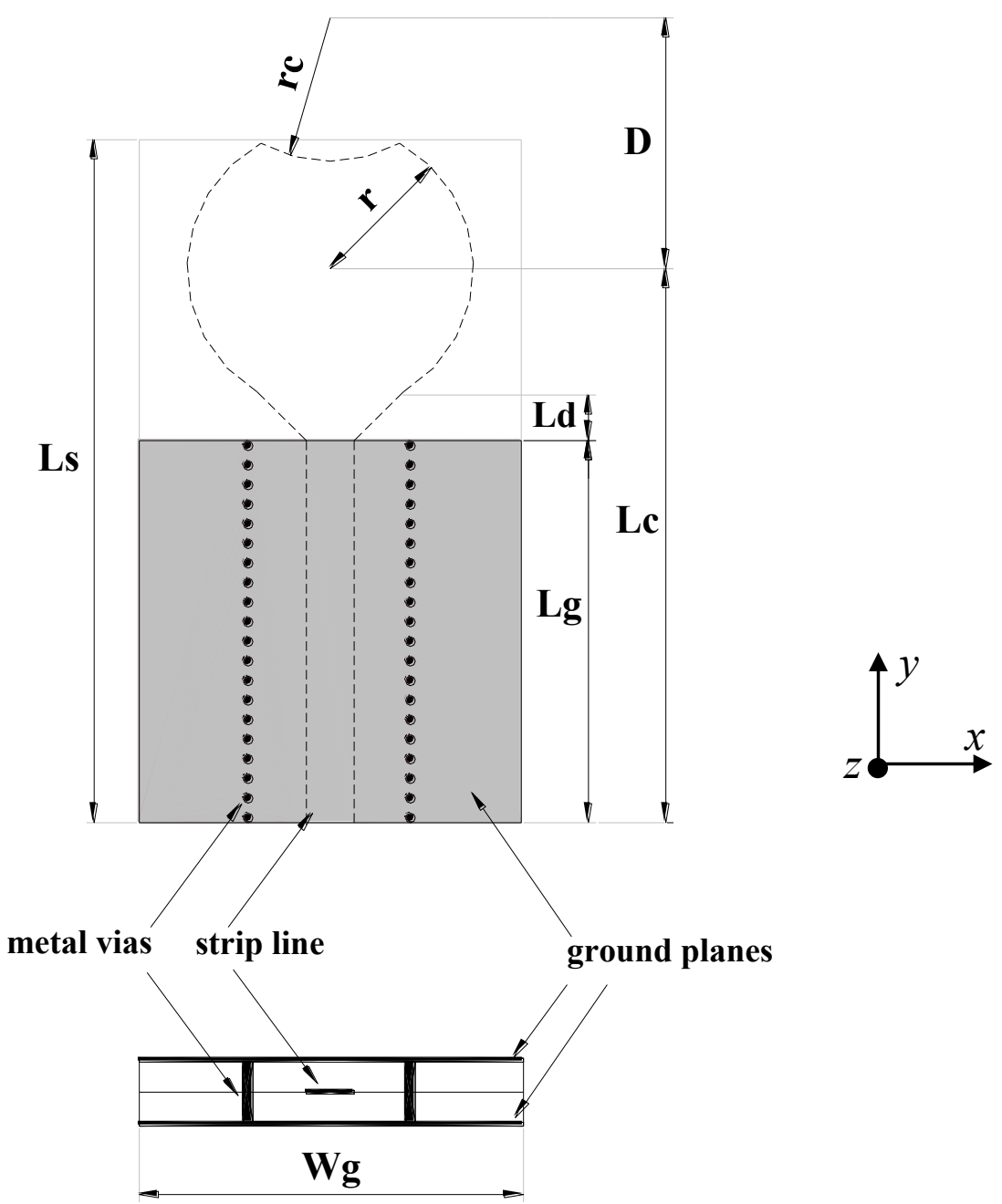

Fig.1. Geometry of the Dual-layer UWB antenna 


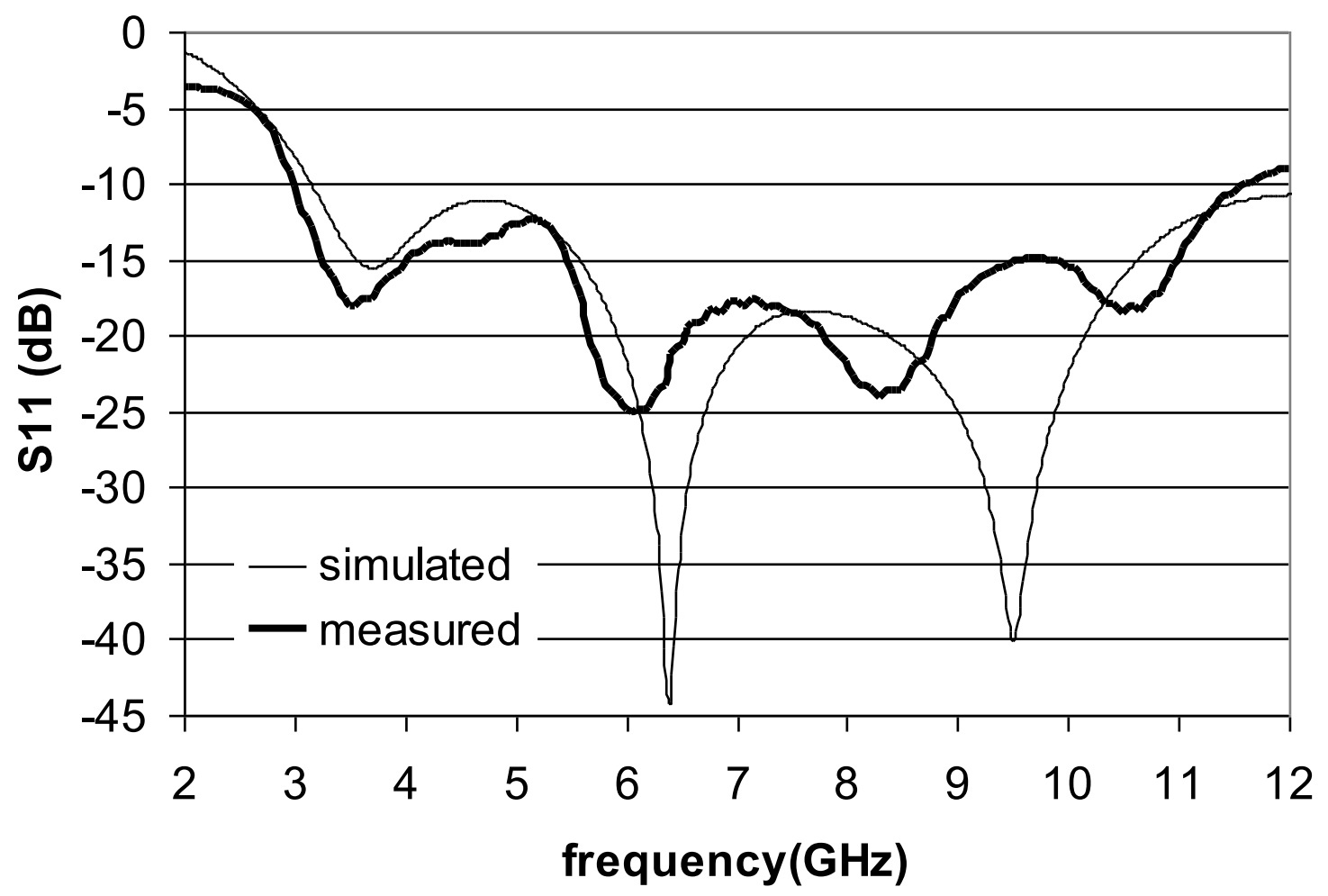

Fig.2. Simulation and measured return loss (S11) of the dual-layer UWB antenna. 


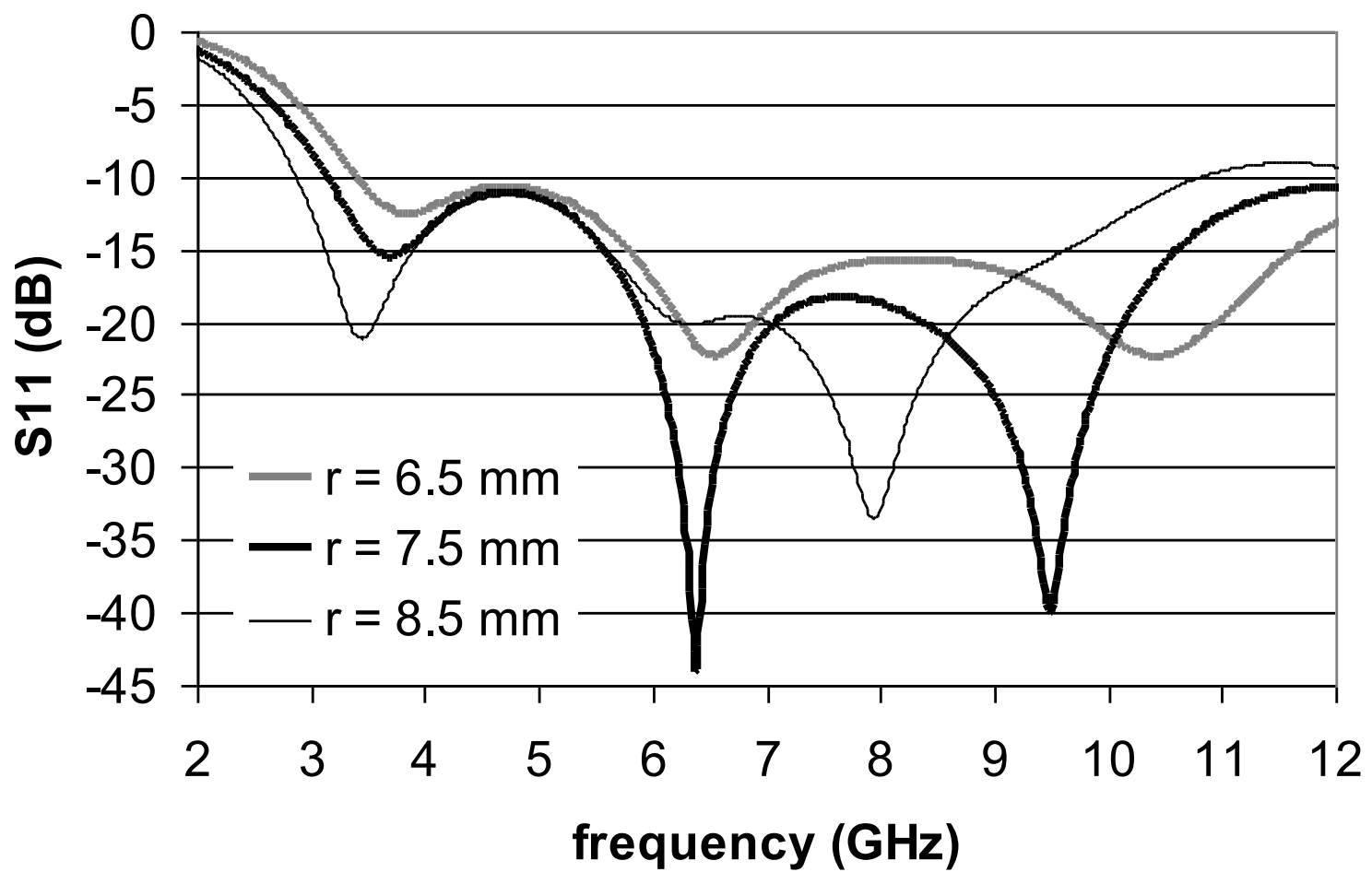

Fig.3. Simulated S11 of the multilayer UWB antenna changing the radius (r) of the circular radiating element. 


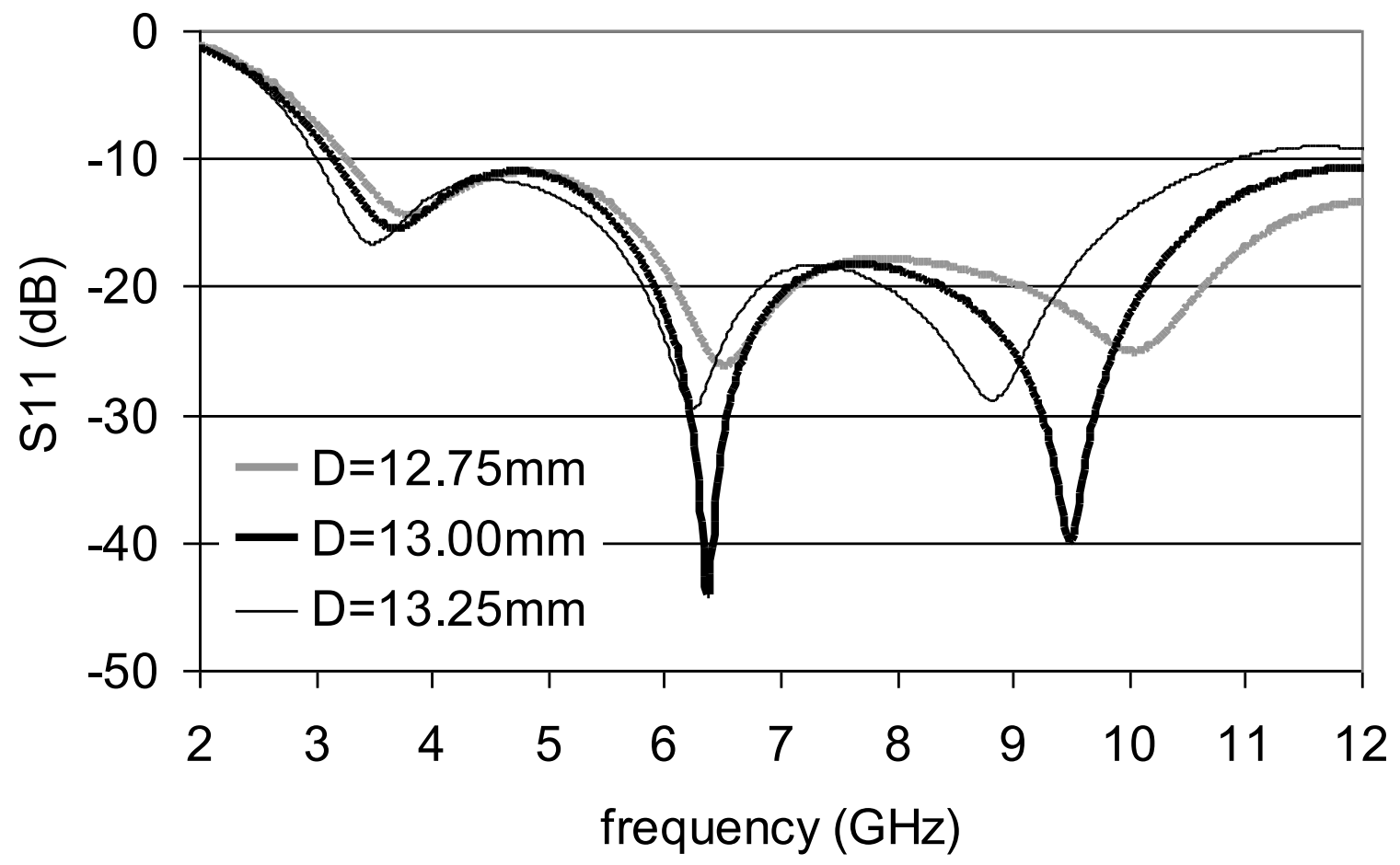

Fig.4. Simulated S11 of the multilayer UWB antenna changing distance from the centre of the radiating disc to the center of the cutting circle (D) 


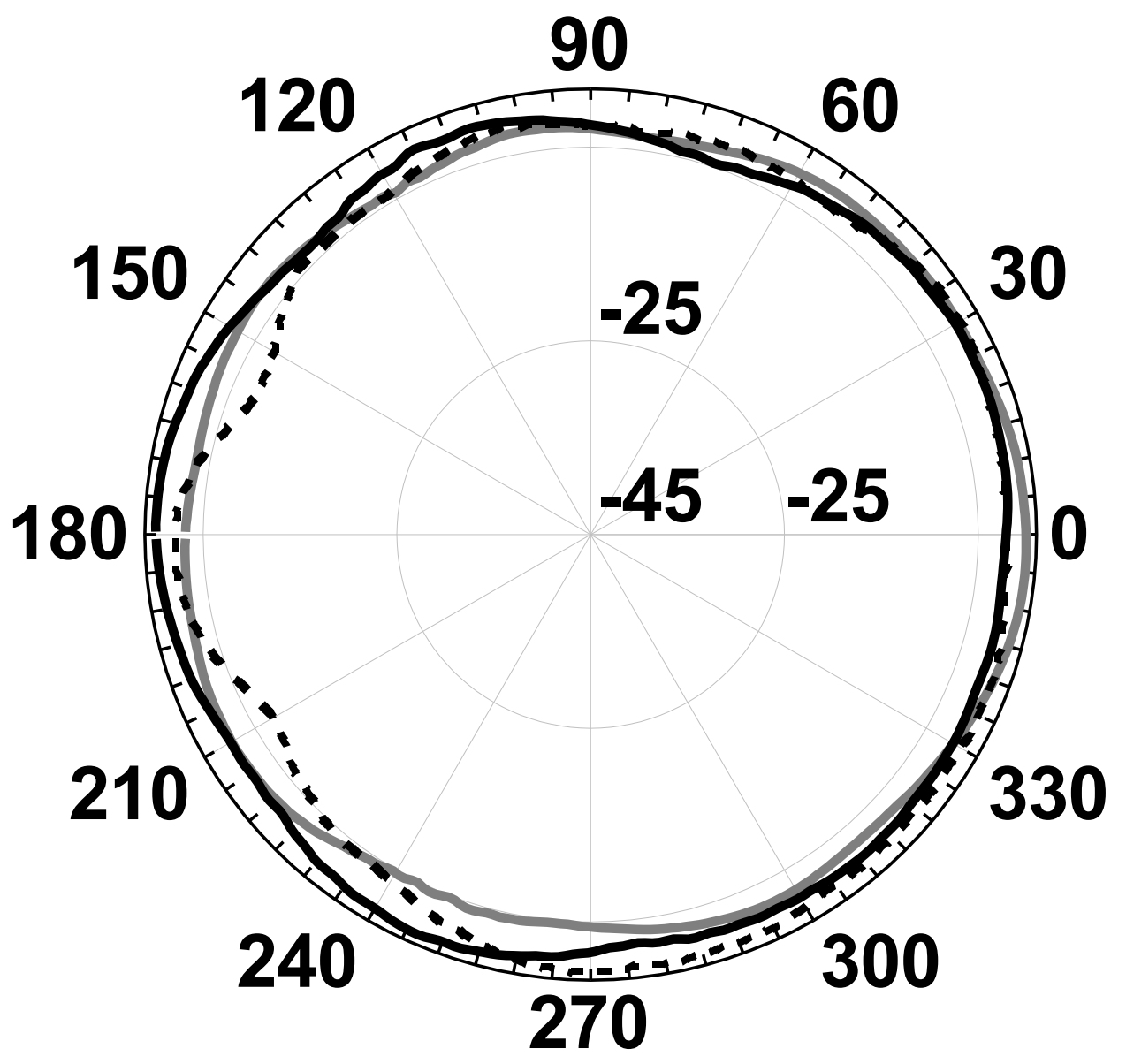

Fig.5. Measured $\mathrm{H}$ plane radiation pattern in the $x-z$ plane at $3.5 \mathrm{GHz}$ (dashed line), $6 \mathrm{GHz}$ (black), $9 \mathrm{GHz}$ (grey). 